\title{
Human optokinetic nystagmus: Competition between stationary and moving displays
}

\author{
CHIEKO M. MURASUGI, LAN P. HOWARD, and MASAO OHMI \\ York University, North York, Ontario, Canada
}

\begin{abstract}
We reported earlier that occlusion of the central retina and stationary edges have highly interactive effects on the gain of optokinetic nystagmus (OKN; Murasugi, Howard, \& Ohmi, 1986). In this study, we explored this effect in more detail. A central occluding band of variable height, flanked by vertical bars, was superimposed onto an array of dots moving at $30^{\circ}$ per second. The height of the occluding band required to abolish OKN increased with the separation of the vertical bars. For bars $3.5^{\circ}$ apart, OKN was abolished in most subjects when a band only $6^{\prime}$ high ran between them. For bars $75^{\circ}$ apart, a band at least $20^{\circ}$ in height was required to abolish the response. The effects of the stationary figure depended to some extent on the subject's attention, but only at intermediate values of bar separation. Both low- and high-level mechaisms are proposed to account for the results.
\end{abstract}

When exposed to a moving display, the eyes automatically execute pursuit movements alternating with return saccades, a response known as optokinetic nystagmus $(\mathrm{OKN})$. Studies of the rabbit, cat, and monkey have revealed that visual inputs feed directly to motion-sensitive cells in the pretectum and are then routed through the vestibular nucleus to the oculomotor system (Collewijn, 1975; Hoffmann \& Distler, 1986; Hoffmann \& Schoppmann, 1981). Optokinetic nystagmus controlled by this primitive system has the following characteristics: (1) it is responsive to the slip velocity of the retinal image, (2) it occurs only in response to temporonasal motion, and (3) it is subject to integral control that maintains pursuit velocity across saccadic interrupts and manifests itself as optokinetic afternystagmus (see Howard, 1982). In carnivores and primates, the pretectum receives supplementary inputs from motion detectors in the visual cortex (Hoffmann, Bauer, Huber; \& Mayr, 1984; Hoffmann \& Schoppmann, 1981). These inputs counteract the directional preponderance of the directly innervated mechanism (Montarolo, Precht, \& Strata, 1981; Ohmi, Howard, \& Eveleigh, 1986; Westall \& Schor, 1985) and allow these animals to pursue selectively one of several conflicting motion signals (Howard \& Gonzalez, 1987). Animals with well-developed fovea have, in addition, evolved the voluntary pursuit and fixation systems that give them the ability to maintain on the fovea the image of a stationary object of interest.

One procedure used to isolate the OKN mechanism from the foveal pursuit system is to place a retinally stabi-

This study was supported by Grant A0195 from the Natural Sciences and Engineering Research Council of Canada awarded to I. P. Howard. Some of the results were presented at the meeting of the Association for Research in Vision and Ophthalmology, Sarasota, Florida, May, 1986. Requests for reprints should be sent to C. M. Murasugi or I. P. Howard, Department of Psychology, York University, 4700 Keele Street, North York, Ontario M3J 1P3, Canada. lized occluder over the central retina. Even small occluders have been reported to produce a large drop in the gain of OKN (eye velocity over stimulus velocity) even at low stimulus velocities (Dubois \& Collewijn, 1979; van Die \& Collewijn, 1982). However, the occluders used in these studies had lateral edges, and, although retinally stabilized, such edges may suppress OKN even when the central retina is not occluded (Howard, Giaschi, \& Murasugi, in press; Wyatt \& Pola, 1984). When Howard and Ohmi (1984) occluded the central retina with a $10^{\circ}$ high horizontal band extending across the whole visual field, they found that the gain of OKN was not reduced significantly at stimulus velocities below about $\mathbf{4 0}$ degrees per second (dps). Van Die and Collewijn (1986) confirmed this finding. All the edges of such an occluder are parallel to the stimulus motion; therefore, any effect of the occluder on $\mathrm{OKN}$ gain must be due only to occlusion. However, although central occlusion has little or no effect on the gain of OKN when there are no lateral edges, occlusion accentuates the suppressive effects of stationary edges (Murasugi et al., 1986). The present experiment was designed to explore this interactive effect.

We know of no other investigators who have systematically examined $\mathrm{OKN}$ suppression when stationary edges were superimposed on a moving display. There have been numerous studies, however, on the ability of subjects to voluntarily pursue targets moving with respect to stationary or moving backgrounds. Kowler, van der Steen, Tamminga, and Collewijn (1984) reported that the ability of subjects to fixate or voluntarily pursue a visual display is virtually unaffected by a superimposed identical moving or stationary display. However, the maximum velocity of stimulus motion in this study was only just over $1 \mathrm{dps}$. Collewijn and Tamminga (1983) found that the addition of a textured background reduced the gain of voluntary pursuit by about $10 \%$ for stimulus velocities of up to about 10 dps. Yee, Daniels, Jones, Baloh, and Hon- 
rubia (1983) reported a similar loss of gain at this velocity, but losses of up to about $20 \%$ for higher velocities. In a later paper, Collewijn and Tamminga (1986) reported that the ability to pursue targets $5^{\circ}$ above and below the fovea was strongly degraded, even at low velocities, by the presence of a stationary structured background. In the present experiment, we were concerned with OKN; we were not interested in the limits to which voluntary pursuit may be pushed. For this reason, subjects were instructed not to pursue particular elements of the OKN display. Of course, the foveation mechanism may well have supplemented $\mathrm{OKN}$ even when subjects made no effort to select particular stimulus elements, especially when the foveal region was not occluded. Indeed, we shall argue that our results may partly be explained in these terms.

The purpose of the present experiment was to investigate the effect on OKN of eccentric stationary edges, alone and in combination with central retinal occlusion. The edges were fixed in space rather than on the retina because we were specifically interested in examining OKN in the presence of competing motion signals. Competition between the mechanisms of object stabilization and background stabilization occurs frequently in the natural environment, but this question has rarely been addressed empirically. Besides the fact that retinally stabilized stimuli do not provide competing motion signals, they differ from space-fixed objects in that they are not often encountered and, when they are, they can by volition be treated as objects that are fixed either in space or on the eye (Howard et al., in press). These properties of retinally stabilized objects make them inappropriate stimuli for use in this experiment. Therefore, we measured the gain of the slow phase of OKN induced by a display of moving dots as a function of (1) the eccentricity of a pair of stationary bars, (2) the height of a horizontal central occluder, and (3) the combined variation of these two factors.

\section{METHOD}

\section{Subjects}

Four adults, including two of the authors, served as subjects. None of the subjects had oculomotor problems, and all had normal or corrected-to-normal eyesight. Two of the subjects were naive about the purpose of the study and had not previously participated in an OKN experiment.

\section{Display}

The optokinetic display consisted of a vertical cylinder of translucent white plastic (2-mm UVEX), $120 \mathrm{~cm}$ in diameter and $75 \mathrm{~cm}$ high. Opaque black dots $2,2.5$, and $3 \mathrm{~cm}$ in diameter were painted at random over the inside of the cylinder, with a mean density of 600 dots per square meter. The display was transilluminated by diffuse tungsten light at a level of $40 \mathrm{~cd} / \mathrm{m}^{2}$ and a contrast of $95 \%$. The cylinder rotated around the subject in either direction at a velocity of $30 \mathrm{dps}$. Pairs of opaque vertical bars symmetrically disposed about the median plane of the subject's head could be placed against the inside of the cylinder. Their inner edges were separated by $3.5^{\circ}, 7.5^{\circ}, 16^{\circ}, 47^{\circ}$, and $75^{\circ}$ of visual angle. The bars with the smallest separation were $12^{\prime}$ wide, and the width of the bars increased approximately in proportion to their eccentricity, thus keeping their visibility roughly constant in terms of the magnification factor of the visual system. The bars extended the full height of the moving display. This enhanced their visibility and kept their height constant. The long bars also provided a convenient support for a horizontal black band, which in some conditions was inserted between them at eye level, as shown in Figure 1d.

Each band had the same curvature as the cylinder and was placed as close as possible to the moving display. The vertical subtense of the band ranged from $9^{\prime}$ to $30^{\circ}$. Bands higher than $30^{\circ}$ were not used because the eye-movement monitor partially blocked the subject's view of the lower visual field, which is important in driving OKN (Murasugi \& Howard, in press).

\section{Procedure}

The subject was seated inside the cylinder with the eyes $57 \mathrm{~cm}$ from the moving display and the head restrained by a dental wax bite. The left eye was covered with an eye patch. We used monocular viewing to eliminate the disparity cues arising from the bars, which were slightly in front of the moving display. The eye-movement monitor was calibrated by asking subjects to fixate targets at known horizontal eccentricities. Eye movements were recorded for about $10 \mathrm{sec}$ after the moving display reached constant velocity. There were four experimental conditions, and the same display of moving dots were used in each. In the band-only condition, horizontal bands between $9^{\prime}$ and $30^{\circ}$ high were displayed in random order one at a time across the center of the moving display, as shown in Figure 1b. In the bars-only condition, the five vertical bars were presented in random order (Figure 1c). In the bars-and-band condition, horizontal bands ranging from $6^{\prime}$ to $30^{\circ}$ in height were inserted in random order between each of the five pairs of vertical bars (Figure 1d). For a given subject and separation of the bars, bands higher than required to suppress OKN completely were not used. In the full-field condition, the moving display had neither bars nor bands (Figure 1a). For each subject, one 10-sec trial was run for each stimulus condition.

The subject was asked to center his or her gaze between the bars and along the midhorizontal axis of the bands. Some of the moving dots at eye level were slightly paler and smaller than the others and



(a) Full field

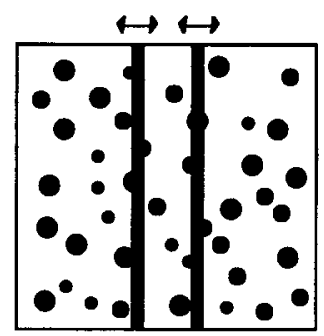

(c) Bars only

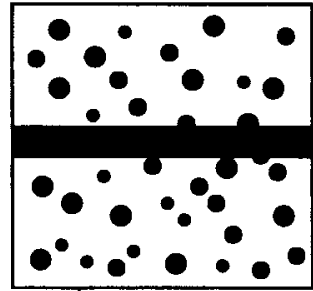

(b) Band only

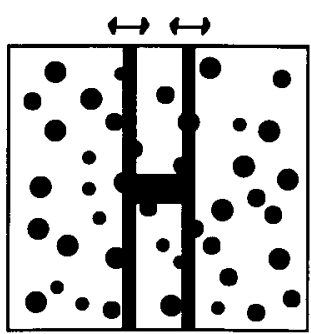

(d) Bars and band
Figure 1. The stimuli used in the four experimental conditions. Stationary vertical bars of various separations and horizontal bands of various heights were superimposed on a display of dots moving across the subject's field of view. 
indicated to the subject where the vertical position of the eyes should be. For each condition, the subject was asked to attend to the moving display in one trial and to the stationary display in another. The order of these instructions was counterbalanced across subjects. In the full-field condition, the subject was instructed to attend to either the moving display or to an imaginary stationary object. The data for each subject were collected in several sessions, with only one session given per day. A full-field condition was run in each session and was used as the baseline measure for other conditions in the same session.

The movements of the subject's right eye were detected by a Biometrics infrared eye-movement monitor with a displacement sensitivity of approximately $15^{\prime}$ of arc. The resulting position signal was sampled and digitized every $10 \mathrm{msec}$ and stored in an Apple computer.

\section{RESULTS}

The mean velocity of the slow phases of OKN during each 10 -sec trial was derived by a computer program that eliminated saccades and blinks. The velocity of the slow phase of OKN evoked by the full-field display in a particular session is designated Fv, and that evoked in the same session when a stationary display is superimposed on the moving display is designated Sv. The suppressive effect of a given stationary display on the velocity of $\mathrm{OKN}$ is indicated by the ratio $\mathrm{Sv} / \mathrm{Fv}$, that is, the ratio of the slow-phase velocity in the presence of the stationary elements to the slow-phase velocity in the absence of the stationary elements. A ratio of 1 indicates that the superimposed elements had no effect on OKN velocity, and a ratio of 0 indicates that the response was totally suppressed by the superimposed display. This ratio will be referred to as the eye-velocity ratio.

The mean retinal eccentricity of the closest of the two vertical bars in each bars-only and bars-and-band ( $9^{\prime}$-wide band only) trial was obtained by using a computer program to calculate the mean eye position during the trial. In addition, the average displacement of the slow phases ( $2 \times$ amplitude) during the same 10 -sec period was obtained by analyzing the eye-movement records by hand.

In Figure 2, the eye-velocity ratios for each subject are plotted for each separation of the bars as a function of the height of the band. The functions on the left were obtained from conditions in which subjects attended to the moving display, and those on the right from conditions in which they attended to the superimposed elements. Eye velocities fluctuated considerably more in all subjects when the bars or band were in view than in the full-field conditions in which $\mathrm{OKN}$ gains were relatively constant. Standard error bars $( \pm 1 S E)$ for each condition are shown for M.S., the subject who exhibited the greatest variability in slow-phase velocity during the trials. The standard errors for the full-field conditions and for the conditions lacking error bars are smaller than the size of the symbols. The mean OKN gains and standard deviations derived from all full-field trials, averaged across the 4 subjects, were, respectively, 1.0 and 0.15 , with the moving display, and 0.94 and 0.19 , with the stationary object.
Note that when we refer to a slow-phase velocity of 0 , we mean 0 with respect to the sensitivity of our eye monitor, namely $42^{\prime}$ per second. The raw data summarized in Figure 2 were analyzed as described in the following three sections.

\section{Effects of Central Occlusion Alone}

In Figure 3, the mean eye-velocity ratio for the 4 subjects is plotted as a function of the height of the occluding band for the two attention instructions in the bandonly conditions. Overall, the OKN response declined with band height.

A two-way (attention $\times$ band height) analysis of variance (ANOVA) with repeated measures performed on the OKN gains from the band-only and full-field conditions revealed a significant effect of band height $[F(5,15)=$ $6.1, p<.05]$ and an attention $\times$ height interaction $[F(5,15)=3.2, p<.05]$. Although there was no main effect of attention, gains were significantly lower when the subject attended to the $5^{\circ}$ and $10^{\circ}$ bands than when they attended to the moving display $\left[5^{\circ}\right.$ band: a posteriori $F(5,18)=14.8, p<.01 ; 10^{\circ}$ band: a posteriori $F(5,18)=23.1, p<.01]$. With the data collapsed over the two attention conditions, the narrowest band $\left(6^{\prime}\right.$ of arc) did not significantly reduce $\mathrm{OKN}$ gain relative to the full-field condition [a posteriori $F(3,12)=2.1$, n.s.]. Bands $5^{\circ}$ and $10^{\circ}$ in height produced a $25 \%$ reduction in gain. The $20^{\circ}$ and $30^{\circ}$ bands had a significantly larger effect [a posteriori $F(5,18)=5.5, p<.01$ ], but even with these bands, the gain of OKN remained over 0.5 .

\section{Effects of Stationary Bars Alone}

The results of the bars-only condition are presented in Figure 4. In Figure $4 a$, the eye-velocity ratios averaged over 4 subjects are plotted as a function of the separation of the vertical bars for each attention condition. The mean retinal eccentricities of the closest bar and the mean slowphase displacements, both averaged across the 4 subjects, are shown for the same experimental conditions in Figure $4 \mathrm{~b}$. According to Figure $4 \mathrm{~b}$, the mean retinal eccentricities of the vertical bars and the mean displacements increased with bar separation, but attention had no systematic effect on eye position or displacement.

It can be seen from Figure 4a that, under both attention conditions, the suppressive effect of the bars became less as they increased in separation or mean retinal eccentricity. Overall, the vertical bars had a greater suppressive effect when subjects attended to them. The effects of bar separation and attention were confirmed by the results of a two-way ANOVA with repeated measures performed on the OKN gains from the bars-only and fullfield conditions [bar separation, $F(5,15)=16.9$, $p<.001$; attention, $F(1,3)=17.4, p<.05]$. There was no significant interaction between separation and attention.

In summary, these results show that stationary objects in the far periphery had little or no effect on OKN even when subjects attended to them. At the other extreme, stationary edges totally or almost totally suppressed OKN 
Subject attends to moving display
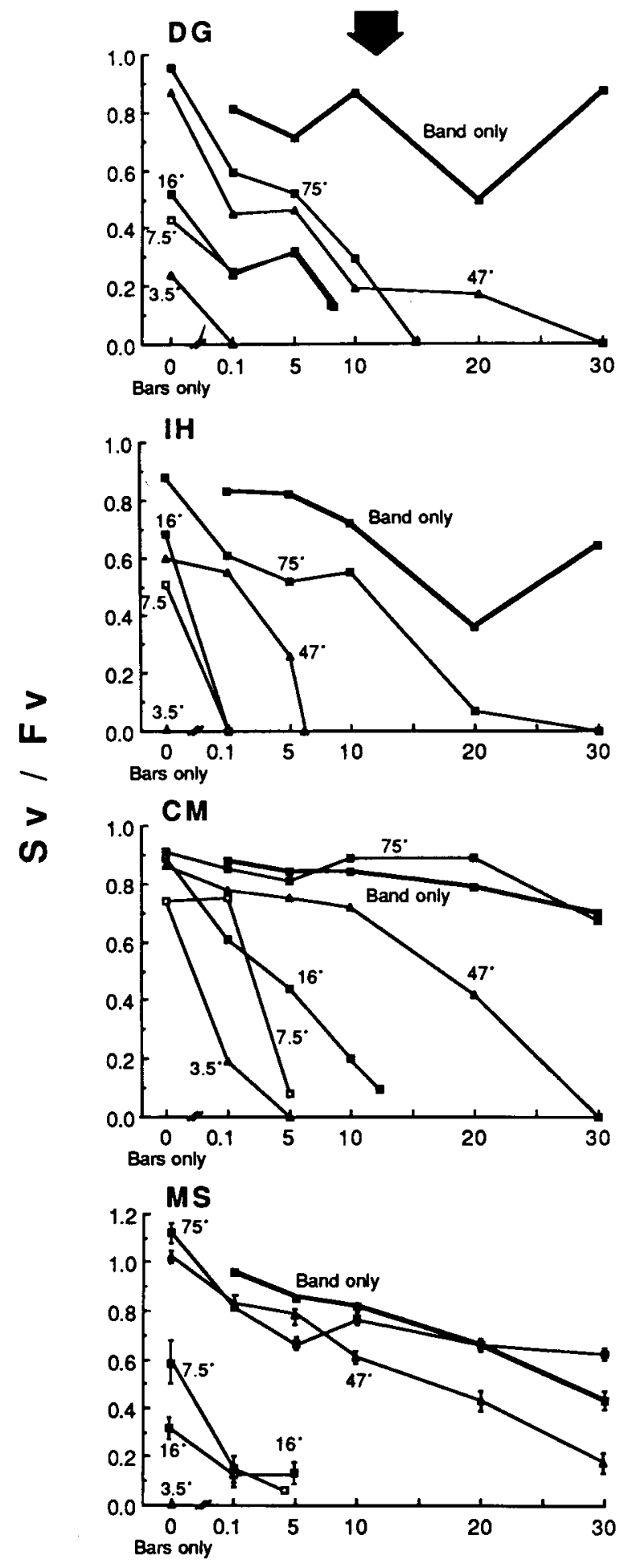

Subject attends to stationary display
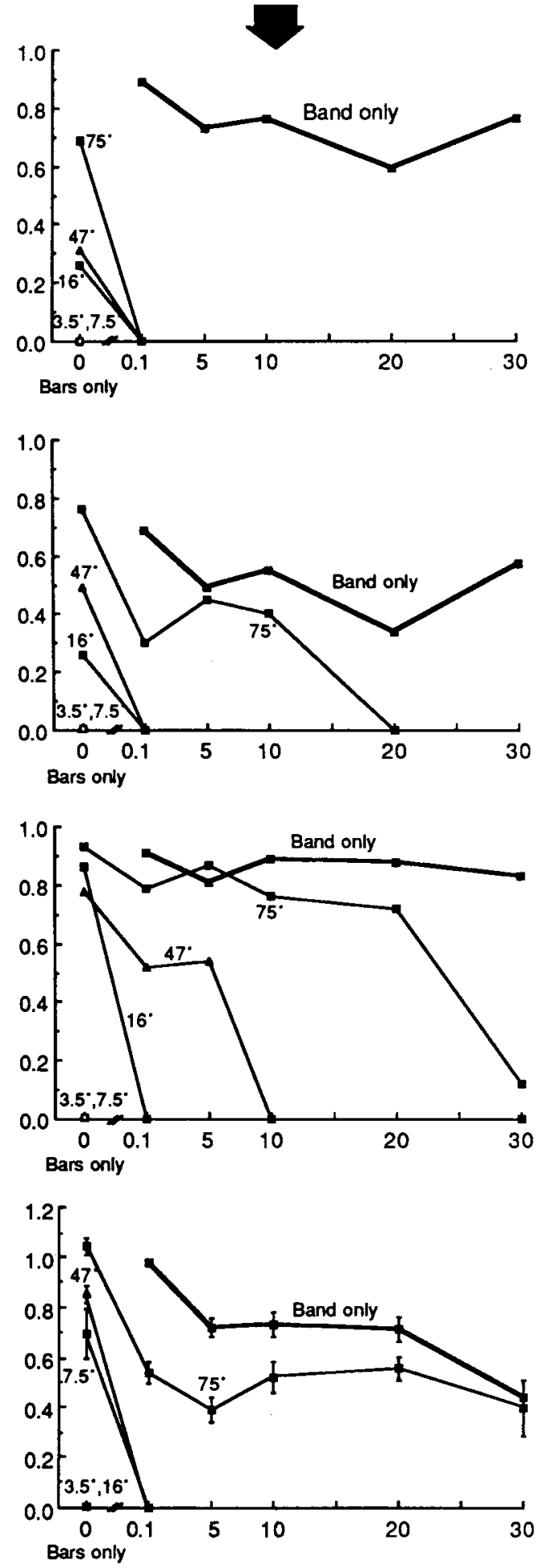

\section{Band height (deg)}

Figure 2. The individual data for 4 subjects under both attention conditions. For each separation of the bars, the ratio of OKN slowphase velocity evoked by the combined stationary and moving displays to that evoked by the full field of moving dots (Sv/Fv) is plotted against the height of the occluding horizontal band. The interrupted line along the abscissa indicates that the distance between zero band height and band heights of $6^{\prime}$ or 9' of arc is overrepresented. Error bars $( \pm 1 S E M)$ are displayed for Subject M.S., who exhibited the greatest variability in slow-phase velocities during the trials. The SEM for the full-field condition and for the conditions lacking error bars are smaller than the size of the symbols. 


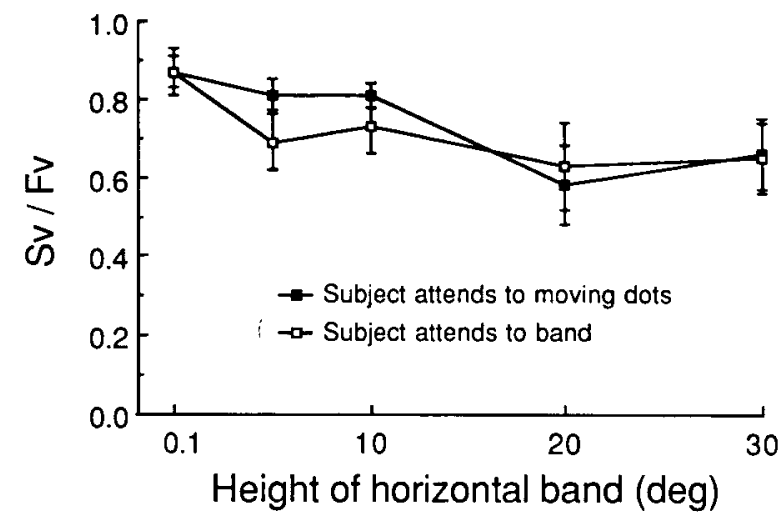

Figure 3. For the band-only conditions, the mean eye-velocity ratios (Sv/Fv) derived from the results of 4 subjects are plotted as a function of the height of the occluding band for each of the attention instructions. Error bars indicate $\pm S E M$.

when placed near the center of the visual field, even when subjects attended to the moving display. We are not claiming that subjects are unable to deliberately pursue elements of a moving display across a stationary object; we claim only that OKN, under the conditions in which it normally manifests itself, does not occur over a centrally placed stationary object.

\section{Combined Effects of Stationary Edges and Central Occlusion}

It is evident from inspection of Figure 2 that the addition of a horizontal band across the stationary vertical bars increased the suppressive effect of the stationary bars. All subjects showed the same general trend: as the eccentricity of the vertical bars increased, the height of the horizontal band required to suppress $\mathrm{OKN}$ by a given amount also increased. In most cases, OKN became totally suppressed when a sufficiently high band was introduced. The band height required to suppress $\mathrm{OKN}$ was equal to about half the separation of the bars in conditions in which the subject attended to the moving display.

The most striking results in the bars-and-band conditions were obtained with the narrowest bands $\left(6^{\prime}\right.$ or $\left.9^{\prime}\right)$. These results are presented in Figure 5. In Figures $5 \mathrm{a}$ and $5 b$, the eye-velocity ratios for the band-only $\left(9^{\prime}\right)$, barsonly, and bars-and-band conditions are plotted as a function of the separation of the vertical bars for the two attention conditions. In Figures $5 \mathrm{c}$ and $5 \mathrm{~d}$, the mean retinal eccentricities of the closest bars are shown for the barsonly and bars-and-band conditions.

The bottom two graphs confirm that the retinal eccentricity of the bars increased with bar separation in a similar manner in the two stimulus conditions, and that eye position did not vary systematically with attention. It is apparent from Figures $5 \mathrm{a}$ and $5 \mathrm{~b}$ that a $9^{\prime}$-wide band had little suppressive effect on its own, but when combined with vertical bars, OKN gains decreased dramatically. This effect was most pronounced when the subject attended to the stationary display.
We also examined the band height required to reduce OKN slow-phase velocity to the lowest measured in the 4 subjects in the bars-and-band conditions. Except for the condition in which a $30^{\circ}$ band was placed between bars $75^{\circ}$ apart, the lowest measured OKN was less than 5 dps. For bars only $3.5^{\circ}$ apart, OKN velocity was never higher than 5 dps when a band only $6^{\prime}$ of arc high was placed across them, even when subjects attended to the moving display. At the other extreme, when the bars were $75^{\circ}$ apart, a band at least $20^{\circ}$ high was required to abolish the response, even when subjects attended to the band. In several cases, even a $30^{\circ}$ band did not eliminate OKN when the bars were $75^{\circ}$ apart (see Figure 2). Overall, subjects differed in the extent to which bands of a given height attenuated the effects of the moving display and in the degree to which these effects were controlled by changes in attention.

We performed a two-way ANOVA with repeated measures to test whether the band height required to abolish or minimize OKN varied with bar separation and/or attention. Both main effects and the interaction were significant [separation, $F(4,12)=13.1, p<.001$; attention, $F(1,3)=16.9, p<.05$; separation $\times$ attention,
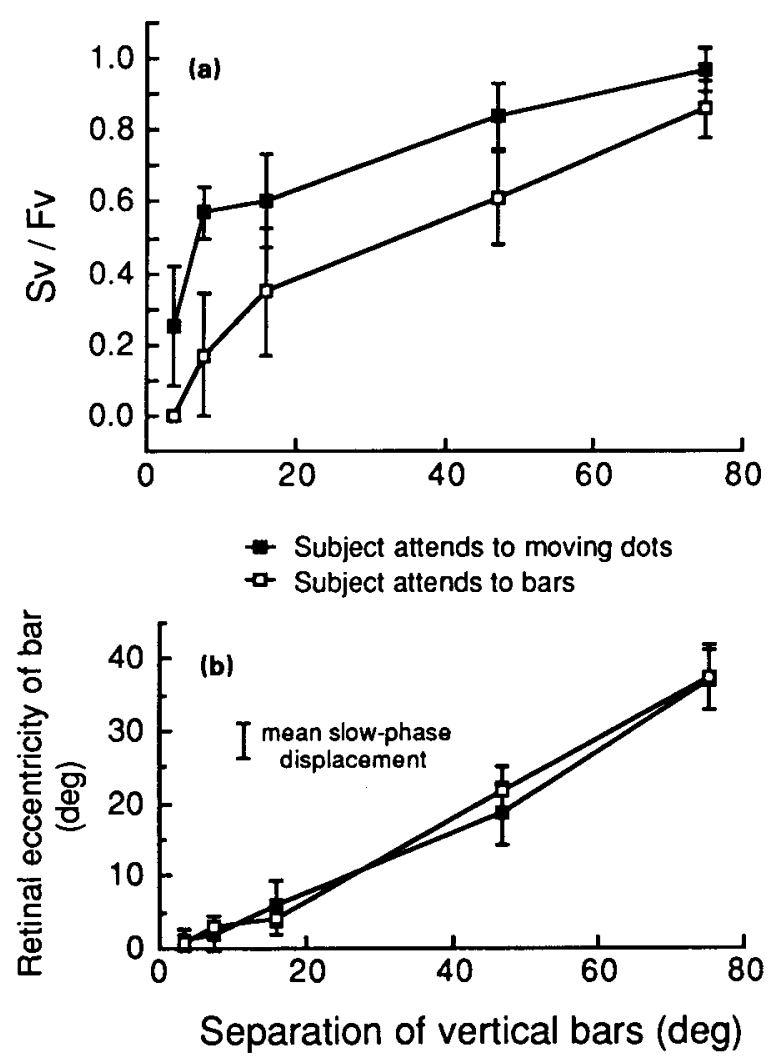

Figure 4. The mean data of 4 subjects for the bars-only conditions. (a) The ratio $\mathrm{Sv} / \mathrm{Fv}$ is plotted against the separation of the vertical bars for each attention instruction. The error bars indicate $\pm 1 S E M$. (b) The mean retinal eccentricities of the closest bar and the mean slow-phase displacements are plotted for the same experimental conditions as those shown in (a). 


\section{Subject attends to moving display}

\section{Subject attends to stationary display}
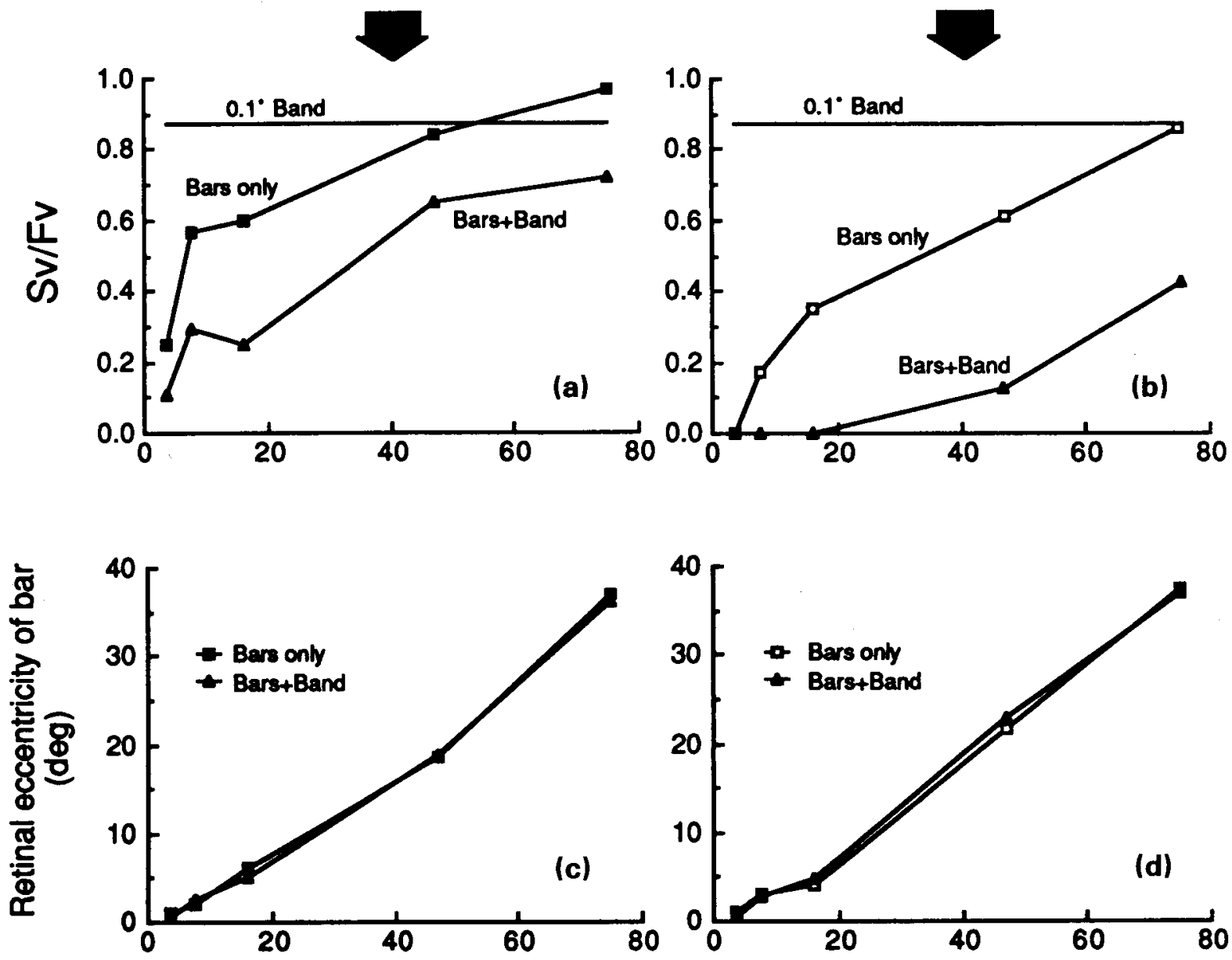

Separation of vertical bars (deg)

Figure 5. A comparison of the mean data of 4 subjects for the bars-only, band-only ( $\left(9\right.$ '-wide band), and bars-and-band ( $6^{\prime}$-wide band) conditions. (a) Eye-velocity ratios for the bars-only and bars-and-band condicions are plotted as a function of the separation of the vertical bars. The eye-velocity ratio obtained with the $9^{\prime}$-wide band is also shown. The subject attended to the moving display. (b) Eye-velocity ratios are plotted for the other attention condition. (c) Mean retinal eccentricities of the closest bars are plotted for the bars-only and bars-and-band conditions as a function of bar separation. The subject attended to the moving display. (d) The mean retinal eccentricities are plotted for the other attention condition.

$F(4,12)=4.6, p<.05]$. The main effect of separation indicated that the band height required to minimize OKN increased with increases in bar separation (see Figure 2). However, the effect of attention was significant only at the $47^{\circ}$ separation [a posteriori $F(1,6)=32.5, p<.01$ ] At this separation, the band height required to suppress OKN was much less when the subjects attended to the stationary display.

\section{DISCUSSION}

\section{Effects of Central Occlusion Alone}

It is difficult to make a direct comparison between the effects of our horizontal band and those of the disk-shaped occluders used by other investigators. However, we can make a direct comparison between the effects of our horizontal bands and those of the vertical bands used by van Die and Collewijn (1982). For instance, van Die and Collewijn found that a $10^{\circ}$-wide vertical band reduced the gain of OKN by more than $50 \%$ at a stimulus velocity of $30 \mathrm{dps}$, whereas our horizontal band reduced it by only $27 \%$ at that velocity, even when the subjects attended to it. The point is that a featureless horizontal band does not generate horizontal motion signals, because its only visible edges run parallel to the direction of motion of the OKN display. In a more recent study, van Die and Collewijn (1986) replicated our earlier findings with horizontal bands and showed that such bands are much 
less suppressive than vertical bands. They attributed the difference in the effects of vertical and horizontal bands to a basic anisotropy in the power of the upper and lower, as opposed to the left and right, hemiretinae to evoke OKN. We believe that the evidence we have reported in this and our earlier publications demonstrates that vertical bands are more suppressive because they contain edges at right angles to the direction of motion. We hypothesize that for vertical $\mathrm{OKN}$, horizontal occluders will be more suppressive than vertical occluders.

It is unlikely that the drop in OKN gain produced by the horizontal bands is due simply to a reduction in the area of the moving display, because quite small moving displays generate high-gain $\mathrm{OKN}$ if centrally placed (Dichgans, 1977). However, the central retina contains a greater density of receptive fields than does the peripheral retina, so that the removal of stimulation from a large number of receptive fields may account for the effects of central occlusion on the gain of OKN. The other possibility is that the loss in gain is due to the absence of the foveal pursuit system when the central retina is occluded. Further work is required to decide between these possibilities.

\section{Effects of Stationary Edges Alone}

When vertical edges alone were superimposed on the moving display, we found that their suppressive effect decreased as a function of their increased eccentricity. These results confirm those published previously (Barnes $\&$ Crombie, 1985; Murasugi et al., 1986). They also are consonant with the report that peripheral targets are pursued less well than central targets over a textured background (Collewijn \& Tamminga, 1986). We conclude that when there are competing motion signals in different parts of the visual field, those motion signals that arise from objects nearest the fovea tend to dominate the oculomotor response. This principle is further illustrated by the fact that when there are two adjacent displays moving in opposite directions, OKN occurs in response to the display upon which the gaze falls. This principle makes functional sense because it is most important for foveate animals to stabilize the images of objects near the center of the visual field.

However, this principle of relative proximity to the central retina cannot explain all our results. According to this principle, there should always have been a vigorous OKN in all conditions of our experiment because the parts of the moving display above and below the band and between the vertical bars were nearer to the fovea than were the stationary edges. Perhaps stationary parts of a display are more potent in controlling eye movements than are moving parts. There is some evidence that, other things being equal, the eyes fixate a stationary display in preference to pursuing a moving one. For instance, Howard and Gonzalez (1987) found that when a moving display and an identical stationary display are superimposed, the eyes do not move when subjects look with relaxed gaze. Of course, the stationary and moving displays used in the present experiment were not identical, so other factors may also have been involved.

\section{Interactive Effects of Central Occlusion and Stationary Edges}

The most interesting aspect of the results of the present experiment is that vertical bars linked by an occluding band had a much greater suppressive effect than bars alone. The result that surprised us most was that two vertical bars, which by themselves had only a small effect on OKN, could suppress the response, sometimes completely, when a horizontal line only $6^{\prime}$ of arc thick was placed between them. As the stationary bars became more eccentric, the horizontal band linking them had to be made higher before OKN was fully suppressed. The effect of adding a linking band was most pronounced when subjects attended to the stationary pattern. The principle of the dominance of stimuli nearer to the fovea explains why, as the stationary bars became more eccentric, the horizontal band linking them had to be made higher before OKN was fully suppressed. In other words, as the vertical bars became more eccentric, their weakened potency to control the oculomotor system could be counteracted if the potency of the moving display was weakened by that display's also being made more eccentric. We argue that, in this situation, occlusion of the central retina affects OKN mainly because it weakens the potency of the moving display relative to the other motion signals.

We think it is unlikely that the effects of placing a band only $6^{\prime}$ of arc thick between two stationary edges can be due entirely to occlusion of the central retina, and we suggest that something like the following may also be happening: When the image of an object traverses the fovea, the visual system looks for edges that will give a clue to its motion. If the object is a horizontal band with no lateral edges, the only motion signals present are those of the moving display. The band and moving display are therefore seen as moving together, and there is relatively little reduction in gain. But if there are stationary vertical edges in view, the band can be seen as part of a stationary coherent display. It is well known that the visual system links together elements of a display that share a common motion (Koffka, 1935; Johansson, 1977; Nakayama, 1985). The retinal motion signals from the bars, when linked by a band, become significant because these motion signals provide the error signal whereby the visual system may stabilize the image of the stationary object. In other words, the relative potency of the bars to gain access to the oculomotor system is increased when they are seen as part of a pattern that crosses the central retina. Whether this increase in potency is enough to suppress $\mathrm{OKN}$ totally will depend on the eccentricity of the bars, the height of the band, and the subject's focus of attention, as has already been explained. 


\section{REFERENCES}

BArnes, G. R., Crombie, J. W. (1985). The interaction of conflicting retinal motion stimuli in oculomotor control. Experimental Brain Research, 59, 548-558.

CollewiJn, H. (1975). Direction-selective units in the rabbit's nucleus of the optic tract. Brain Research, 100, 489-508.

Collewijn, H., \& Tamminga, E. P. (1983). Human smooth and saccadic eye movements during voluntary pursuit of different target motions on different backgrounds. Journal of Physiology, 351, 217-250.

Collewij, H., \& Tamminga, E. P. (1986). Human fixation and pursuit in normal and open-loop conditions: Effects of central and peripheral retinal targets. Journal of Physiology, 379, 109-129.

Dichgans, J. (1977). Optokinetic nystagmus as dependent on the retinal periphery via the vestibular nucleus. In R. Baker \& A. Berthoz (Eds.), Control of gaze by brain stem neurons (pp. 261-267). Amsterdam, The Netherlands: Elsevier/North Holland.

Dubois, M. F. W., \& Collewis, H. (1979). Optokinetic reactions in man elicited by localized retinal motion signals. Vision Research, 19, $1105-1115$.

Hofrmann, K. P., Bauer, R., Huber, H. P., \& Mayr, M. (1984). Single cell activity in area 18 of the cat's visual cortex during optokinetic nystagmus. Experimental Brain Research, 57, 118-127.

Hoffmann, K. P., Distler, C. (1986). The role of direction selective cells in the nucleus of the optic tract of cat and monkey during optokinetic nystagmus. In E. L. Keller \& D. S. Zee (Eds.), Adaptive processes in visual and oculomotor systems (pp. 261-266). Oxford: Pergamon.

Hoffmann, K. P., \& SchoppmanN, A. (1981). A quantitative analysis of the direction-specific response of neurons in the cat's nucleus of the optic tract. Experimental Brain Research, 42, 146-157.

HowARD, I. P. (1982). Human visual orientation. Chichester: Wiley.

Howard, I. P., Giaschi, E., \& Murasugi, C. M. (in press). Suppression of OKN and VOR by afterimages and imaginary objects. $E x-$ perimental Brain Research.

HowaRD, I. P., \& Gonzalez, E. G. (1987). Human optokinetic nystagmus in response to moving binocularly disparate stimuli. Vision Research, 27, 1807-1816.

Howard, I. P., \& OHMI, M. (1984). The efficiency of the central and peripheral retina in driving human optokinetic nystagmus. Vision Research, 24, 969-976.
Johansson, G. (1977). Studies on visual perception of locomotion. Perception, 6, 365-376.

KoFFKA, K. (1935). Principles of gestalt psychology. New York: Harcourt Brace.

Kowler, E., van der Steen, J., Tamminga, E. P., \& Collewiun, H. (1984). Voluntary selection of the target for smooth pursuit in the presence of superimposed, full-field stationary and moving stimuli. Vision Research, 24, 1789-1798.

Montarolo, W., Precht, W., Strata, P. (1981). Functional organization of the mechanisms subserving optokinetic nystagmus in the cat. Neuroscience, 6, 231-246.

Murasugi, C. M., \& Howard, I. P. (in press). Human horizontal optokinetic nystagmus elicited by the upper versus the lower visual fields. Visual Neuroscience.

Murasugi, C. M., Howard, I. P., \& OHMI, M. (1986). Optokinetic nystagmus: The effects of stationary edges, alone and in combination with central occlusion. Vision Research, 26, 1155-1162.

Nakayama, K. (1985). Biological image motion processing: A review. Vision Research, 25, 625-660.

Ohmi, M., Howard, I. P., \& Eveleigh, B. (1986). Directional preponderance in human optokinetic nystagmus. Experimental Brain Research, 63, 387-394.

van Die, G., \& Collewun, H. (1982). Optokinetic nystagmus in man Human Neurobiology, 1, 111-119.

VAN DiE, G., \& CollewiJn, H. (1986). Control of human optokinetic nystagmus by the central and peripheral retina: Effects of partial visual field masking, scotopic vision and central retinal scotomata. Brain Research, 383, 185-194.

Westall, C. A., SCHOR, C. M. (1985). Asymmetries of optokinetic nystagmus in amblyopia: The effect of selected retinal stimulation. Vision Research, 25, 1431-1438

WyatT, H. J., Pola, J. (1984). A mechanism for suppression of optokinesis. Vision Research, 24, 1931-1945.

Yee, R. D., Daniels, S. A., Jones, O. W., Baloh, R. W., HoNRUBIA, V. (1983). Effects of optokinetic background on pursuit eye movements. Investigative Ophthalmology \& Visual Science, 24, 1115-1122.

(Manuscript received April 15, 1988; revision accepted for publication August $9,1988$. ) 